are highly effective anticonvulsants against seizures induced in animals by a variety of mechanisms including kindling. Adenosine receptor antagonists exert proconvulsant actions indicating tonic adenosinergic control of ictal susceptibility. Animal studies have shown a rapid and substantial release of adenosine and its metabolites inosine and hypoxanthine in brain interstitial fluid during experimental seizures. ${ }^{2}$ The physiological actions and metabolism of adenosine in the human CNS remain to be fully elucidated. We report that acute increases of adenosine metabolites can be detected in lumbar CSF after clinical status epilepticus in humans.

Seven patients with new onset status epilepticus were studied. Five patients (aged 12 to 67) developed generalised status epilepticus with three or more clonic or tonic-clonic seizures occurring in less than one hour without regaining consciousness between seizures. Causes included viral encephalitis, cysticercosis, uraemia, benzodiazepine withdrawal, and drug toxicity. One patient (48 years old) with diabetes and chronic renal failure developed epilepsia partialis continua involving repeated clonic seizures of the right face and arm. One patient (82 years old) experienced complex partial status epilepticus documented by electroencephalography due to withdrawal from alprazolam. Eleven patients (aged 22 to 53) evaluated for other neurological conditions (demyelinating neuropathy, headaches, cranial neuropathy, multiple sclerosis) were used as controls.

All CSF samples used in this study were discarded specimens from lumbar punctures performed for diagnostic purposes as part of each patient's clinical evaluation. The CSF was refrigerated at less than $4^{\circ} \mathrm{C}$. Cell counts ranged from 0-30 white blood cells $/ \mathrm{mm}^{3}$ and 0-92 red blood cells $/ \mathrm{mm} .^{3}$

Nanomolar concentrations of adenosine were determined by high performance liquid chromatographic-fluorometric assay as described by Zhang et al. ${ }^{3}$ Micromolar concentrations of nucleosides and purine bases (for example, adenosine, inosine, and hypoxanthine) were determined by high performance liquid chromatographic assay using a Beckman Ultrasphere ODS $4.6 \times$ $250 \mathrm{~mm}$ column eluted at $1.0 \mathrm{ml} / \mathrm{min}$ at ambient temperature with a gradient of buffer A $(20 \mathrm{mM}$ potassium phosphate $\mathrm{pH}$ $3.5)$ and buffer $B$ (acetonitrile) (0-1 min, $0 \%$; $1-18 \mathrm{~min}, 0-4 \%$; $18-28 \mathrm{~min}, 4-7 \%$; 28-33 min, 7-80\% buffer B) and detection by ultraviolet absorbance at $254 \mathrm{nM}$.

Concentrations of adenosine in CSF obtained from control patients without seizures were in the low nanomolar range whereas concentrations of the adenosine metabolites inosine and hypoxanthine were considerably higher (table). These values are comparable with those reported by other investigators. ${ }^{4}$ The concentrations of adenosine and its metabolites in lumbar CSF most likely reflect brain interstitial concentrations as the relative concentrations of these purine compounds in control samples are almost identical with those measured in the interstitial fluid from the frontal cortex of animals. ${ }^{2}$ The low concentrations of adenosine cannot be attributed to ex vivo degradation as there is no appreciable metabolism of adenosine in CSF. ${ }^{4}$

Five patients evaluated for generalised convulsive status epilepticus underwent diagnostic lumbar punctures $0 \cdot 1$ to 13 hours after termination of their last seizure. No changes in CSF adenosine concentrations were found but inosine and hypoxanthine concentrations were substantially higher than controls (table). The time interval from the last seizure to CSF sampling seemed to be an important factor with the maximum increase of hypoxanthine (greater than sixfold) seen at one hour. Nevertheless, increases in inosine and hypoxanthine concentrations were still evident up to 13 hours. Patient 4 had another diagnostic lumbar puncture 12 days after recovery from her last seizure and all adenosine metabolites had returned to concentrations comparable with controls (adenosine $<0.039 \mu \mathrm{M}$, inosine $0.65 \mu \mathrm{M}$, hypoxanthine $2 \cdot 31 \mu \mathrm{M})$. A sample of CSF from patient 6 with recurrent simple partial seizures showed a doubling in adenosine compared with the mean control value with a minimal increase in hypoxanthine. Patient 7 underwent lumbar puncture during continuous complex partial status epilepticus and a modest increase in adenosine and inosine but not hypoxanthine was found.

This study is the first to document rapid and substantial changes in adenosine metabolites in CSF after status epilepticus in humans. Our data complement the recent findings of During and Spencer ${ }^{5}$ who reported profound increases in extracellular fluid adenosine from the hippocampus in four patients with complex partial seizures, using microdialysis probes attached to depth electrodes. Our findings also closely parallel the reported changes in adenosine metabolites in interstitial fluid after experimental seizures in animals. For example, Park $e t a l^{2}$ found that extracellular adenosine concentrations rose significantly during 10 minutes of bicuculline induced seizures in paralysed, ventilated piglets. Smaller increases in inosine and hypoxanthine were found during the same period. Ten minutes

Cerebrospinal fluid concentrations of adenosine and metabolites after status epilepticus

\begin{tabular}{|c|c|c|c|c|c|}
\hline & Seizure & Time(h) & $\begin{array}{l}\text { Adenosine } \\
(\mu M)\end{array}$ & $\begin{array}{l}\text { Inosine } \\
(\mu M)\end{array}$ & $\begin{array}{l}\text { Hypoxanthine } \\
(\mu M)\end{array}$ \\
\hline $\begin{array}{l}\text { Controls }(\mathrm{n}=11) \\
\text { (mean (range)) } \\
\text { Patients: }\end{array}$ & - & - & $\begin{array}{l}0.063 \\
(0.039-0.106)\end{array}$ & $\begin{array}{l}0.41 \\
(\mathrm{ND}-0.79)\end{array}$ & $\begin{array}{l}2 \cdot 36 \\
(1 \cdot 27-4 \cdot 08)\end{array}$ \\
\hline 1 & GTC & $<1 \cdot 0$ & 0.050 & 1.47 & $12 \cdot 42$ \\
\hline 2 & GTC & $1 \cdot 0$ & 0.040 & 1.91 & $15 \cdot 78$ \\
\hline 3 & GTC & $4 \cdot 0$ & 0.061 & ND & $15 \cdot 39$ \\
\hline 4 & G'TC & $9 \cdot 0$ & 0.072 & $2 \cdot 47$ & $8 \cdot 20$ \\
\hline 5 & GTC & $13 \cdot 0$ & 0.027 & 0.71 & $4 \cdot 34$ \\
\hline 6 & EPC & - & $0 \cdot 120$ & ND & $3 \cdot 20$ \\
\hline 7 & CPSE & - & $0 \cdot 102$ & $1 \cdot 33$ & 1.98 \\
\hline
\end{tabular}

ND = none detected; GTC = generalised tonic-clonic status epilepticus; EPC = epilepsia partialis continua; CPSE = complex partial status epilepticus; time = estimated time from last seizure to sampling of CSF. Patient 7 was sampled during continuous seizure activity. Patient 6 had multiple simple partial motor seizures and time from last seizure is not available.

after cessation of seizures, adenosine concentration returned to preictal values whereas inosine and hypoxanthine continued to rise, indicating that adenosine released interstitially during seizures is rapidly metabolised. Our findings of similar increases in inosine and hypoxanthine concentrations but not adenosine in patients after generalised status epilepticus can be explained by such rapid metabolism of adenosine in vivo.

The physiological relevance of our findings to the regulation of epileptic phenomena in humans is unknown. Extensive experimental data in animals indicate that adenosine and its metabolites are important endogenous modulators of seizure initiation and propagation. ${ }^{1}$ Whether similar mechanisms are operative in humans remains to be determined.

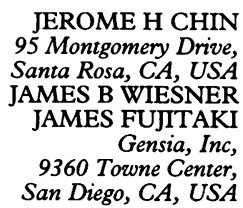

Correspondence to: $\mathrm{Dr}$ Jerome $\mathrm{H}$ Chin, 95 Montgomery Drive, Suite 102, Santa Rosa, CA 95404, USA.

$1 \mathrm{Chin} \mathrm{JH}$. Adenosine receptors in brain: neuromodulation and role in epilepsy. Ann Neurol 1989;26:695-8.

Park TS, Van Wylen DGL, Rubio R, Berne RM. Interstitial fluid adenosine and sagittal sinus blood flow during bicuculline-seizures in newborn piglets. $f$ Cereb Blood Flow Metab 1987;7:633-9.

3 Zhang Y, Geiger JD, Lautt WW. Improved high-pressure liquid chromatographic-fluosine in plasma. Am f Physiol 1991;260: G658-64.

4 Ohisalo JJ, Murros K, Fredholm BB, Hare TA. Concentrations of gamma-aminobutyric acid and adenosine in CSF in progressive myoclonus epilepsy without Lafora's bodies. Arch Neurol 1983;40:623-5.

5 During MJ, Spencer DD. Adenosine: a potential mediator of seizure arrest and posticta refractoriness. Ann Neurol 1992;32:618-24.

Bilateral cavernous sinus thrombosis causing Korsakofr's amnesic syndrome

Korsakoff's amnesic syndrome (Korsakoff's psychosis) is a condition in which learning and memory are affected out of proportion to other cognitive functions. It has not been previously reported as a complication of cavernous sinus thrombosis. Our patient developed Korsakoff's amnesic syndrome after bilateral medial temporo-occipital infarcts as a complication of cavernous sinus thrombosis.

A 44 year old man was admitted with fever of 15 days duration, and altered sensorium of 10 days duration preceded by a day of recurrent vomiting. Three days before admission he developed painful swelling of his left eye associated with redness and sudden total loss of vision in that eye. The fever was high grade, intermittent, and associated with sweating. The altered sensorium was in the form of restlessness, incoherent speech, disorientation, and an inability to recognise his relatives properly. He had had hypertension for four years duration, was on irregular treatment, and was a past smoker. He had no history of alcohol intake.

On examination he was drowsy, febrile $\left(99^{\circ} \mathrm{F}\right)$, and had a blood pressure of 


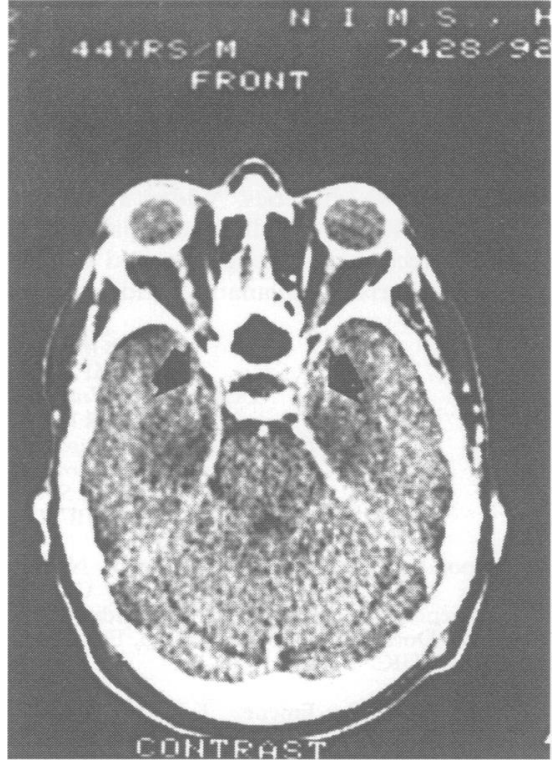

Figure 1 Contrast CT showing bilateral medial temporo-occipital infarctions with filling defects and convex bulging of the lateral walls (arrows) of the cavernous sinuses.

134/90 mm Hg. General and systemic examinations were otherwise unremarkable. Examination showed a proptosed left eye, circumcorneal congestion, absent perception of light, and direct light reflex with a pale optic disc; findings on the right side were normal except for a sluggish direct light reflex. Other cranial nerves were normal. Motor system examination was unremarkable. A mild neck rigidity was present. The next day the left sided proptosis was more prominent and associated with chemosis, pronounced lid oedema, and severe restriction of all movements of the left eyeball. The intraocular pressure was 40 $\mathrm{mm} \mathrm{Hg}$ on the left side and $14 \mathrm{~mm} \mathrm{Hg}$ on the right.

Relevant laboratory data included mild normocytic, normochromic anaemia $(\mathrm{Hb}-$ $12 \cdot 1 \mathrm{~g} / \mathrm{dl}$ ) with mild neutrophilia and a normal platelet count with normal concentrations of blood glucose, creatinine, serum urea nitrogen, sodium, and potassium. Liver function tests, prothrombin and activated partial thromboplastin times, blood culture, ECG, chest radiograph, and two dimensional echocardiogram were all normal. A CSF examination showed normal pressure, clear acellular fluid with $77 \mathrm{mg} / \mathrm{dl}$ of sugar, and $50 \mathrm{mg} / \mathrm{dl}$ of protein.

Plain head CT showed bilateral asymmetric medial temporo-occipital infarctions, the left being larger. Repeat CT two days later with contrast showed enhancement of the cavernous sinus with filling defects and bulging of the lateral margins of both cavernous sinuses (fig 1) - more so on the left, and the infarcts seemed more prominent. The paranasal sinuses were normal.

The patient was treated with cefotaxime and amikacin for the next two weeks, with intravenous mannitol and local application of steroids and atropine for the eyes. Anticoagulants were not given. He became apyrexial with gradual improvement of his sensorium. A third plain CT two weeks after admission showed bilateral haemorrhagic transformation of the infarcts, more prominent in the left medial temporooccipital area.
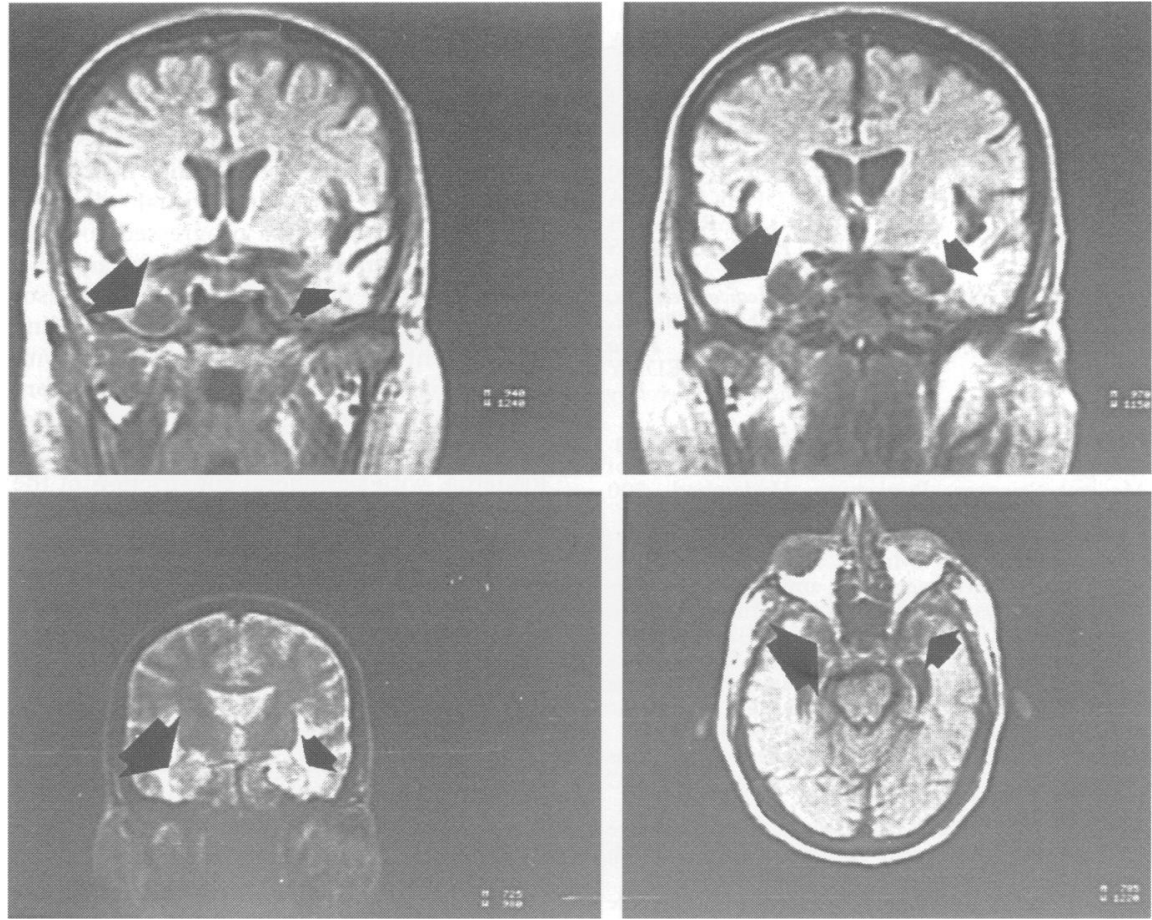

Figure 2 Coronal T1 (top row) and T2 (bottom left) weighted and axial T1 (bottom right) MRI slices showing bilateral temporal polar infarcts with enlargement of the temporal horns (arrows: large-right, small-left). The left eyeball is hyperintense and atrophic.

A detailed mental status examination three weeks after admission showed that attention was mildly abnormal with mild anomia in naming objects and parts of objects; the remaining language functions were essentially normal. $\mathrm{He}$ was disoriented for time, place, and person. Immediate and remote memories were mildly impaired with gross recent memory (both verbal and visual new learning abilities) impairment. Momentary confabulations were noted with normal constructional abilities. Abstraction was impaired with partially preserved insight and a grossly normal fund of information. Mild impairment of the frontal lobe (inability to do alternating motor patterns) and occipital dysfunction (prosapagnosia and a colour agnosia) were seen. Parietal lobe tests were normal.

Follow up 15 months after discharge showed a persistent confabulatory amnesic state with total blindness of his left eye. The right eye was normal. Visual evoked potentials were normal on the right side but absent on the left. He was unable to return to work or function independently due to the continued severity of his memory impairment.

An MRI at this time showed bilateral well delineated temporal infarcts, which were hypointense in $\mathrm{T} 1$ and hyperintense in T2 images (fig 2). There was evidence of bilateral temporal atrophy with dilatation of the temporal horns of the lateral ventricles. The left eyeball was atrophic.

A neuropsychological assessment 18 months after the onset showed that on the Weschler memory scale his raw score was 22 and his Lui weighted score was 64 . Overall memory quotient was 69. Functional analysis indicated that disturbances persisted in all the memory functions, with recent and immediate memory the worst.

Korsakoff's amnesic syndrome after cav- ernous sinus thrombosis has not been reported before. Damage to three relatively discrete neuroanatomical regions has been primarily implicated in the amnesic syndrome-namely, the medial temporal lobes, the medial thalamic nuclei, and the basal forebrain. Mishkin's model proposes that the degree of impairment of recognition memory was a direct function of the amount of conjoint damage to the amygdala and hippocampus irrespective of the specific structure involved. ${ }^{1}$ In this case the bilateral medial temporo-occipital infarcts also resulted in bilateral hippocampal damage together with amygdalar involvement (fig 2), which is consistent with Mishkin's model for amnesia. This finding is consistent with the hippocampal abnormalities in amnesic patients as shown by high resolution MRI ${ }^{2}$

Clinicopathologically this patient had infarctions bilaterally in the territory drained by the basal vein of Rosenthal (BVR). The BVR receives tributaries from the insula, cerebral peduncles, medial temporal lobe, and veins of the temporal horn. It communicates with the cavernous sinus either through the sphenoparietal sinus ${ }^{3}$ or through the superior petrosal sinus, via the lateral mesencephalic vein. In instances of hypoplastic posterior segment of the BVR, the vein drains anteriorly via the lateral mesencephalic vein. In this patient the thrombotic process may have extended to the BVRs by any one of these channels or, alternatively, the BVRs may have had hypoplastic posterior segments. The diencephalic structures, drained by the internal cerebral veins, were spared in this patient as was the basal forebrain, confirmed by MRI.

Levine et $a l^{5}$ recommend early anticoagulant treatment in cavernous sinus thrombosis to reduce morbidity, but the spontaneous haemorrhagic conversion as seen in our patient suggests that adequate 
imaging either with CT or MRI to rule out mesial temporal infarcts should be mandatory before giving anticoagulant treatment.

We thank Dr K Niranjan Reddy for help in the neuropsychological assessment of the patient. RUPAM BORGOHAIN HARI RADHAKRISHNA AJAY KUMAR SINGH SURATH MOHANDAS Department of Neurology, Nizam's Institute of Medical Sciences, Punjagutta, Hyderabad-500 482,

J JAGANMOHAN REDDY Department of Imageology

Correspondence to: Dr Rupam Borgohain, Department of Neurology, Nizam's Institute of Medical Sciences, Punjagutta, Hyderabad-500 482, India.

1 Saunders RC, Murray EA, Mishkin M. Further evidence that amygdala and hippocampus contribute equally to recognition

2 memory. Neuropsychologia 1984;22:785cass GA, Amaral DG, Squire LR. Hippocampal abnormalities in amnesic patients revealed by high resolution magnetic

3 Stephens RB, Stilwell DB. Arteries and veins Stephens RB, Stilwell DB. Arteries and veins
of the human brain, Springfield, IL: CC of the human brain,
Thomas, 1969:124-67.

4 Babin E, Megret M. Variations in the drainage of the basal vein. Neuroradiology 1973;6: 154-61.

5 Levine SR, Twyman RE, Gilman S. The role of anticoagulation in cavernous sinus thrombosis. Neurology 1988;38:517-22.

\section{Hearing loss as a false localising sign in raised intracranial pressure}

Benign intracranial hypertension (BIH) is an idiopathic disorder characterised by headache and visual disturbances with papilloedema (unilateral or bilateral), in which a space occupying lesion or infective processes have been excluded by neuroimaging, analysis of CSF, and additional ancillary investigations. Recently this fournal has carried a series of reports describing rare associated cranial nerve palsies. ${ }^{1-3}$ We report a case of left sided hearing loss occurring in conjunction with worsening symptoms of raised intracranial pressure in a patient with established BIH that resolved after lumbar puncture.

In December 1993, a 24 year old woman presented to the eye casualty department with a week's history of headache-worse on bending - nausea with intermittent vomiting, and visual blurring. She had been taking oral contraceptives but there was no other relevant drug or medical history. Examination showed obesity (95 kg/155 $\mathrm{cm}$ ), bilateral papilloedema, and normal visual acuity although testing fields by confrontation showed a small central scotoma affecting the right eye and decreased temporal fields in the left eye. Computed tomography, brain MRI, and MR angiography were normal. Lumbar puncture produced clear and colourless CSF with an opening pressure of $36 \mathrm{~cm}$ CSF. The CSF constituents were normal, with a protein concentration of less than $0.1 \mathrm{~g} / \mathrm{l}$ and 2 white cells per cubic mm. The CSF glucose to plasma ratio was normal. The following peripheral blood indices were also normal: full blood count, viscosity, urea and electrolytes, liver function tests, thyroid functions, creatine, protein, venereal disease research laboratory test, and anticardiolipin antibody. OKP-Humphrey field analysis showed pronounced peripheral field constriction. The initial management was withdrawal of the oral contraceptive pill. Diuretic treatment with chlorthalidone was commenced and dietetic referral arranged.

These measures initially resulted in considerable symptomatic improvement. After several months, however, the headaches recurred with increasing visual obscurations. These symptoms progressed to daily bilateral obscurations of vision for up to a minute despite diuretic treatment. In association with this she had noted increasing impairment of hearing in the left ear, with no associated tinnitus, in the week before readmission. Repeat neurological examination on readmission showed chronic papilloedema, with peripheral constriction of the fields but normal visual acuity. Neuro-otological examination confirmed clinical hearing loss in the left ear. The Weber test lateralised to the right. The Rinne test was positive bilaterally. Pure tone audiometry showed a mild left sided conductive hearing loss, with an air bone gap ranging between 5 and $20 \mathrm{~dB}$ at all frequencies tested $(250$ to $4000 \mathrm{~Hz}$ ). Tympanometry showed normal middle ear pressure and compliance bilaterally. Repeat lumbar puncture subsequently confirmed raised pressure of $29 \mathrm{~cm}$ CSF. On the day after the lumbar puncture the patient noted normalisation of the hearing acuity in the left ear. Repeat audiometry and tympanometry were performed showing resolution of the previously noted mild conductive hearing loss and unchanged tympanometry.

Abducens nerve palsies are described in between $9 \%$ and $36 \%$ of cases of benign intracranial hypertension as false localising signs. Other cranial nerve palsies occurring with this disorder are rare, but oculomotor, trochlear, trigeminal, and facial nerve lesions have recently been reported. ${ }^{1-3}$ It has been suggested that potential mechanisms for these occurrences are direct compression of the nerve root by cerebral tissue, traction of the nerve by caudal displacement of the brainstem, or vascular disturbance as a consequence of either of the above. ${ }^{3}$

In this case there was not only a close relation between the worsening symptoms of raised intracranial pressure and the development of left sided hearing loss but also rapid normalisation of hearing acuity on reduction of the raised intracranial pressure, suggesting that the hearing loss may have been a pressure related phenomenon. In the current case, however, the audiometric pattern was indicative of a conductive hearing loss. A potential explanation is to infer an increase in the perilymphatic fluid pressure transmitted through the cochlear aqueduct as a result of the rise in CSF pressure. This might dampen the movement of the stapedial footplate and of the round window membrane giving a small conductive hearing loss. The alternative explanation of a temporary coexistent middle ear effusion is unlikely given the normal tympanometry.

Although otological manifestations have been previously reported in raised intracranial pressure of varying aetiologies including benign intracranial hypertension, ${ }^{4-5}$ hearing loss is not normally considered in the context of false localising signs. It may be under recognised given the typically mild nature of the hearing loss ${ }^{5}$ and the wide prevalence of hearing loss in the normal population.

In a series of 20 patients with benign intracranial hypertension the commonest otological manifestations were objective pulsatile tinnitus and low frequency hearing loss. ${ }^{4}$ Both these symptoms improved transiently in all patients after lumbar puncture. In the longer term these symptoms responded well to weight reduction and treatment with diuretics. Wider awareness of this association will allow such patients to avoid unnecessary investigation and benefit from appropriate explanation and reassurance.

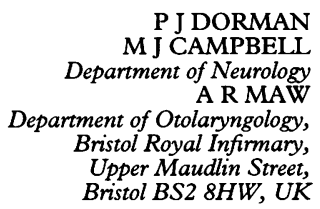

Correspondence to: $\operatorname{Dr} P$ J Dorman, Neurosciences Trials Unit, Department of Clinical sciences Trials Unit, Department of Clinical Neurosciences, University of Edinburgh, BH4 2XU, UK

1 Zachariah SB, Jimenez L, Zachariah B, Prockop LD. Pseudotumour cerebri with focal neurological deficit. $\mathcal{F}$ Neurol Neurosurg Psychiatry 1990;53:360-1.

2 Davie C, Kennedy P, Katifi HA. Seventh nerve palsy as a false localising sign. $\mathcal{f}$ Neurol Neurosurg Psychiatry 1992;55:510-1.

3 Davenport RJ, Will RG, Galloway PJ. Isolated intracranial hypertension presenting with trigeminal neuropathy. $₹$ Neurol Neurosurg Psychiatry 1994;57:381.

4 Sismanis A. Otological manifestations of benign intracranial hypertension syndrome: diagnosis and management. Laryngoscope diagnosis and

5 Tandon PN, Sinha A, Kacker RK, Saxena RK, Singh K. Auditory function in raised intracranial pressure. $\mathcal{F}$ Neurol Sci 1973;18: 455-67.

Bromate intoxication with polyneuropathy

Acute bromate intoxication is a rare event in neurological clinics. Previous reports described renal failure and hearing deficit in addition to nausea, vomiting, haemolytic anaemia, depressed consciousness, and seizure. ${ }^{1}$ We report a woman who attempted suicide by taking a hair permanent wave preparation. This resulted in acute bromate intoxication and she developed renal failure, deafness, and toxic polyneuropathy.

The 25 year old woman (body weight $57 \mathrm{~kg}$ ) took $7.5 \mathrm{~g}$ sodium bromate in the suicide attempt in 1992. Nausea, vomiting, and diarrhoea developed rapidly. Stomach lavage and irrigation by activated charcoal were carried out at a local hospital. Tinnitus and dizziness occurred five hours later, followed by deafness. Blood urea nitrogen was $10 \mathrm{mg} / \mathrm{dl}$ (normal 7-20 mg/dl) and serum creatinine $1.4 \mathrm{mg} / \mathrm{dl}$ (normal $0.5-1.2 \mathrm{mg} / \mathrm{dl}$ for women). Oliguria was noted on the second day, with blood urea nitrogen and creatinine rising to $16 \mathrm{mg} / \mathrm{dl}$ and $3 \mathrm{mg} / \mathrm{dl}$ respectively. The urine sediment disclosed 3-5 red blood cells per high power field (normal 0-2), and 45-50 white blood cells per high power field (normal $<5$ per high power field). Proteinuria was present (protein $>3 \mathrm{mg} / \mathrm{dl}$ but without casts). Serum bromide $\left(\mathrm{Br}^{-}\right)$concentration was $85 \mu \mathrm{g} / \mathrm{ml}$ (none present in normal subjects). Oliguria was resistant to furosemide. On the third day, serum urea nitrogen reached $48 \mathrm{mg} / \mathrm{dl}$ and creatinine $9.3 \mathrm{mg} / \mathrm{dl}$. Because of deteriorating renal function she was referred to the Veterans General Hospital and received three courses of haemodialysis. 